\title{
Skin Granuloma
}

National Cancer Institute

\section{Source}

National Cancer Institute. Skin Granuloma. NCI Thesaurus. Code C36195.

A granuloma located in the skin. 